\title{
Florescimento precoce em pessegueiro e sua relação com a temperatura: um estudo de caso
}

\author{
Augusto Carlos Pola ${ }^{1}$, Emílio Della Bruna ${ }^{1}$, Henrique Belmonte Petry² e Alexsander Luis Moreto ${ }^{2}$
}

\begin{abstract}
Resumo - Em 2013 foi registrada uma floração precoce e intensa em pessegueiros do município de Urussanga, SC, Brasil. Vinte por cento dos acessos de uma coleção de pessegueiros apresentaram plena floração em meados de abril, quando normalmente esta ocorre nos meses de julho e agosto. As faixas térmicas com temperaturas entre 16,0 e $19,0^{\circ} \mathrm{C}$ e inferiores a $19,0^{\circ} \mathrm{C}(13,0$ a $19,0^{\circ} \mathrm{C}$ ), em um período de 49 dias entre fevereiro e março, foram as que mais se relacionaram com a antecipação da plena floração com correlações de -0,92 ( $p<0,01)$ e -0,85 ( $p<0,02)$, respectivamente. Embora as temperaturas entre 16,0 e $19,0^{\circ} \mathrm{C}$ não sejam consideradas efetivas para a endodormência em muitos dos modelos fenológicos tradicionais, alguns trabalhos indicam que, em genótipos com baixa exigência em frio, estas podem desempenhar tal função. Entretanto, assumindo-se uma acumulação simultânea de frio e calor, como proposto por modelos paralelos de quebra de dormência, não é possível afirmar se, no presente caso, essa faixa térmica teve, realmente, um efeito vernalizante.
\end{abstract}

Termos de indexação: Prunus persica; endodormência; floração; modelos fenológicos.

\section{Premature flowering in peach trees and its relation with temperature: a case study}

\begin{abstract}
In 2013, a premature and intense flowering was recorded in peach trees in the city of Urussanga, SC, Brazil. Twenty percent of the accesses of a peach tree collection showed full bloom in mid-April, when it usually only occurs in July and August. Thermal ranges with temperatures between 16.0 and $19.0^{\circ} \mathrm{C}$ and below $19.0^{\circ} \mathrm{C}\left(13.0\right.$ to $\left.19.0^{\circ} \mathrm{C}\right)$, in a period of 49 days between February and March, were the most related with the anticipation of the full bloom, with correlations of $-0.92(p<0.01)$ and $-0.85(p<0.02)$, respectively. Although temperatures between 16.0 and $19.0^{\circ} \mathrm{C}$ are not considered effective for endodormancy in many of the traditional phenological models, some studies indicate that, in genotypes with low chilling demand, they can perform this function. However, assuming a simultaneous accumulation of cold and heat, as proposed by parallel models of dormancy breaking, it is not possible to state whether this thermal range really had a vernalizing effect in this case.
\end{abstract}

Index terms: Prunus persica; endodormancy; flowering; phenological models.

\section{Introdução}

O florescimento do pessegueiro ocorre após a planta ter superado a sua fase de dormência, período no qual as gemas, para se desenvolverem normalmente e atingirem a antese, necessitam satisfazer o seu requerimento fisiológico de baixas temperaturas e de calor. A fase de dormência das frutíferas de clima temperado é comumente subdividida em três estágios: paradormência, endodormência e ecodormência (LANG et al., 1987). Estes estágios variam em termos de duração e intensidade, dependendo principalmente de fatores genéticos e meteorológicos. A temperatura é considerada o principal elemento climático relacionado às atividades fisiológicas deste período de repouso.
No estágio da paradormência, o sinal bioquímico para o controle do crescimento origina-se fora da estrutura (gema) afetada. Este sinal bioquímico pode ser causado por fatores externos, do ambiente, como o fotoperíodo, ou internos, da planta, como a dominância apical de uma gema terminal sobre as laterais ou das folhas sobre as gemas. $\mathrm{Na}$ endodormência o controle do crescimento ocorre somente dentro da estrutura afetada. Na ecodormência são fatores ambientais que restringem o desenvolvimento (HRUY et al., 2013), como estiagens e temperaturas extremas.

Os diferentes tipos de dormência podem atuar simultaneamente, mas a sequência típica, da senescência até a brotação/floração é geralmente a paradormência seguida pela endodormência e, após, a ecodormência (BONHOMME et al., 2000). De maneira geral, a planta sai da paradormência à medida que as folhas caem, e entra na endodormência sob a influência de baixas temperaturas. Segundo Yamane (2014), é difícil diferenciar os efeitos da paradormência e da endodormência sobre as gemas das frutíferas de clima temperado.

No ano de 2013, observou-se a ocorrência de uma floração intensa e anormalmente precoce em pessegueiros de Urussanga, SC. Cerca de $20 \%$ das variedades de uma coleção de pessegueiros apresentaram florescimento abundante no mês de abril, sendo que normalmente a plena floração é registrada nos meses de julho e agosto. No presente estudo procurou-se determinar quais os principais níveis e limites térmicos relacionados a esta floração precoce. São também apresentadas hipóteses e dis-

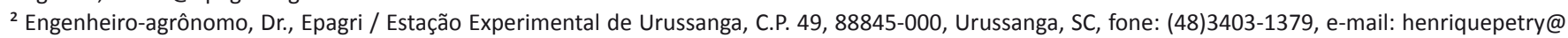
epagri.sc.gov.br; alexsandermoreto@epagri.sc.gov.br. 
cutidos outros aspectos envolvidos com esta anomalia.

\section{Material e métodos}

Foram utilizados, no presente estudo, os registros das datas de plena floração dos acessos da coleção de pessegueiro da Epagri, localizada em Urussanga, estado de Santa Catarina, Brasil, latitude $28^{\circ} 31^{\prime} \mathrm{S}$, longitude $49^{\circ} 19^{\prime} \mathrm{W}$ e altitude de $49 \mathrm{~m}$. Esta coleção é composta por seleções promissoras, provenientes de um projeto de melhoramento genético para as plantas do estado, com predominância de genótipos com baixa exigência em frio. Foram utilizados registros fenológicos dos anos de 2007 a 2013. A data de plena floração foi considerada aquela em que as plantas apresentavam $70 \%$ de flores abertas, sendo determinada visualmente. A distância entre a coleção e a estação meteorológica é de aproximadamente 200 metros.

O clima da região é subtropical úmido, com verão quente e sem estação seca definida (Cfa, segundo a classificação de Köppen). Na Tabela 1 são apresentadas algumas médias mensais de alguns elementos meteorológicos que representam o clima da região do estudo.

Os valores diários de temperatura média, máxima, mínima e temperatura de bulbo seco das 9:00 h e das 21:00 h de 2007 a 2016 foram coletados na estação meteorológica convencional na estação experimental de Urussanga, pertencente ao Instituto Nacional de Meteorologia (Inmet) e operada pela Empresa de Pesquisa Agropecuária e Extensão Rural (Epagri), instalada nas coordenadas de latitude $28^{\circ} 31^{\prime} \mathrm{S}$, longitude $49^{\circ} 19^{\prime} \mathrm{W}$ e altitude de $49 \mathrm{~m}$. O número de horas diárias em que a temperatura permaneceu entre duas temperaturas base foram estimados através da temperatura horária. As temperaturas horárias foram estimadas por interpolação linear simples, considerando uma variação linear entre os horários de ocorrência das quatro temperaturas registradas diariamente (temperatura mínima, temperatura do bulbo seco das 9:00 h, temperatura máxima e temperatura do bulbo seco das 21:00 h) e considerado fixo o horário de ocorrência das temperaturas extremas (6:00 e 15:00 h).

Foi estimado o total de horas em que as temperaturas horárias permaneceram em oito faixas térmicas pré-determinadas:

$(T \leq 7 ; i<T \leq i+3, i \in\{7,10,13,16,19,22\} ; T>25)$ O tempo de permanência da temperatura nestas faixas foi calculado com a utilização de planilha eletrônica e de funções condicionais.

Para a construção dos histogramas da ocorrência da plena floração em pessegueiro por decêndio no período de 2007 a 2012, assim como nos estudos de correlação entre a data de plena floração da "seleção 205" de pessegueiro e os totais de horas acumulados de 11 de fevereiro até determinadas datas, foram utilizados somente os registros de plantas com mais de três anos de idade, visando diminuir a influência da juvenilidade sobre os resultados.

\section{Resultados e discussão}

A plena floração do pessegueiro, em Urussanga, normalmente ocorre no inverno, entre os meses de julho e agosto, como pode ser observado na Figura $1 \mathrm{~A}$. Entretanto, no ano de 2013 foi observado um florescimento outonal anômalo em cerca de $20 \%$ dos acessos da coleção de pessegueiros da Epagri, na cidade. A plena floração, no referido ano, ocorreu em meados de abril em $18 \%$ dos acessos e, no início de maio, em 2\% deles (Figura 1B). A menor concentração de flores abertas foi observada na parte superior e externa das plantas, onde o desfolhamento foi mais lento. Estas flores precoces abortaram e os genótipos voltaram a florescer novamente no inverno, mas com menor intensidade. A brotação de gemas vegetativas não ocorreu fora da época normal.

No mês de abril, normalmente os pessegueiros de Urussanga ainda mantém as suas folhas. Uma abscisão foliar natural e gradual ocorre de março a maio. Porém, no ano de 2013, ocorreu um ataque intenso de ferrugem (Tranzschelia discolor), que antecipou e concentrou o período de queda das folhas. Neste ano, o mês de fevereiro apresentou uma precipitação elevada $(389,7 \mathrm{~mm})$, com 21 dias de chuva, e as plantas da coleção não receberam tratamento antifúngico. Em razão deste ataque severo de ferrugem, as plantas já se encontravam desfolhadas em meados de abril, época em que foi observada a plena floração. A queda prematura de folhas, principalmente quando causada por problemas fitossanitários, induz a um florescimento antecipado, que poderá ocorrer durante o outono, sobretudo em cultivares de baixa necessidade de frio, diminuindo o número de gemas viáveis no final do inverno (SCARIOTTO, 2011). Uma desfolhação precoce afeta a profundidade de dormência e a capacidade de crescimento das gemas, mas o quanto estes fatores afetam o desenvolvimento das gemas depende do cultivar (LLOYD \& FIRTH, 1990). Mesmo em um clima tropical, a endodormência ocorre nos pessegueiros, embora com leve intensidade e por um curto período, e a liberação desta condição pode ocor-»

Tabela 1. Temperatura média (T med), média das temperaturas máximas (T max), média das temperaturas mínimas (T min) e precipitação total $(\mathrm{P})$ em diferentes meses referentes a 30 anos de registros da Estação Meteorológica de Urussanga, SC, Brasil

Table 1. Mean temperature (Tmed), mean maximum temperatures (Tmax), mean minimum temperatures (Tmin) and total precipitation (P) in different months referring to 30 years of records of Urussanga Meteorological Station, SC, Brazil

\begin{tabular}{lccccccccccccc}
\hline & Jan & Fev & Mar & Abr & Mai & Jun & Jul & Ago & Set & Out & Nov & Dez \\
\hline $\mathrm{T}_{\operatorname{med}}\left({ }^{\circ} \mathrm{C}\right)$ & 23,6 & 23,6 & 22,6 & 19,9 & 16,9 & 15,0 & 14,4 & 15,5 & 17,0 & 19,0 & 20,8 & 22,6 \\
$\mathrm{~T} \max \left({ }^{\circ} \mathrm{C}\right)$ & 30,1 & 29,8 & 28,9 & 26,5 & 24,1 & 22,2 & 22,0 & 22,8 & 23,5 & 25,1 & 27,0 & 29,2 \\
$\mathrm{~T} \min \left({ }^{\circ} \mathrm{C}\right)$ & 18,5 & 18,8 & 17,8 & 14,8 & 11,7 & 9,7 & 9,0 & 10,0 & 11,9 & 13,9 & 15,4 & 17,2 \\
$\mathrm{P}(\mathrm{mm})$ & 198,8 & 201,2 & 156,8 & 104,3 & 95,3 & 85,5 & 91,9 & 106,9 & 129,2 & 131,2 & 121,2 & 147,0 \\
\hline
\end{tabular}

Fonte: EPAGRI /CIRAM (2018) 
rer mesmo sob temperaturas amenas (BALANDIER et al., 1993; BALANDIER et al., 1995). Uma dormência profunda é associada a baixas temperaturas, requerendo mais horas de frio para quebrar a dormência do que naquelas com dormência mais superficial (SHERMAN \& LYRENE,1984). Portanto, as plantas que apresentaram florescimento precoce em abril de 2013 provavelmente entraram no estágio de endodormência, permanecendo em um nível superficial.

Quanto maior for o frio acumulado pelas gemas, mais precocemente ocorrerá a data de plena floração e menor será a necessidade de calor para o florescimento (OKIE \& BLACKBURN, 2011a; LI et al., 2016). Modelos de unidades de frio desenvolvidos para o pessegueiro, como o modelo de Utah (RICHARDSON et al., 1974) ou modificações deste (MELKE, 2015), consideram somente temperaturas abaixo de $13,0^{\circ} \mathrm{C}$ como efetivas para a acumulação de frio. Em 2013, em Urussanga, ocorreram somente duas horas com temperaturas abaixo de $13,0^{\circ} \mathrm{C}$ até 10 de abril. Nesse sentido, pode-se considerar que temperaturas abaixo deste nível térmico não tiveram influência sobre a antecipação da floração naquele ano.

$\mathrm{Na}$ Tabela 2, apresentamos o somatório decendial de horas em que a temperatura permaneceu entre 13,0 e $16,0^{\circ} \mathrm{C}$ no período de 1 ㅇ de janeiro a 10 de abril dos anos de 2007 a 2016. Como pode ser observado na tabela, os totais acumulados de horas em que a temperatura permaneceu neste nível térmico, até o dia 10 de abril, no ano de 2013, são próximos ou inferiores aos valores acumulados em outros anos. Pode-se deduzir, portanto, que este nível térmico, ao ser analisado isoladamente, não explica o florescimento precoce registrado. Entretanto, como pode ser constatado na Tabela 3, o número de horas com temperaturas entre 16,0 e $19,0^{\circ} \mathrm{C}$ acumuladas no referido ano é superior ao dos demais anos. Assim, esta faixa térmica, mesmo que considerada de maneira isolada, pode estar associada ou ter sido responsável pela floração anormalmente precoce que foi registrada em 2013.

Na Figura 2, está apresentado o resultado de correlações entre temperaturas em determinadas faixas térmicas e as datas de plena floração de uma
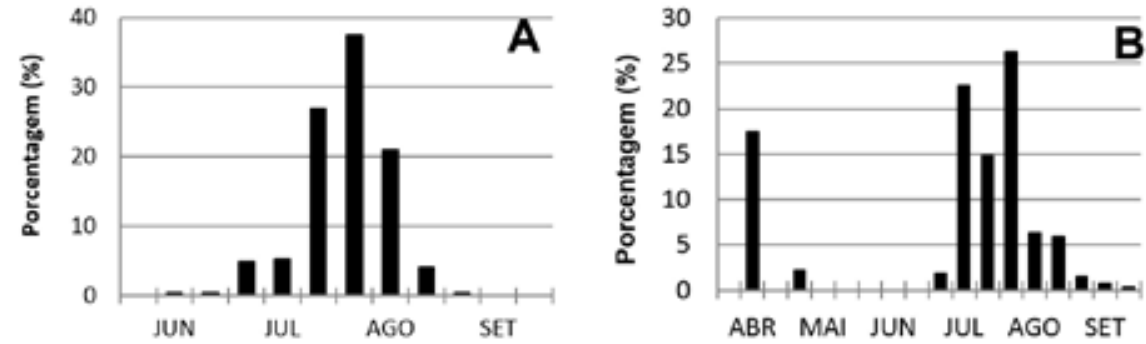

Figura 1. Histogramas representativos da ocorrência da plena floração em pessegueiro por decêndio de 2007 a 2012 (A) e em 2013 (B) na coleção de pessegueiro da Estação Experimental de Urussanga, localizada no sul do estado de Santa Catarina. Os gráficos são resultantes de 783 e 266 registros fenológicos, respectivamente

Figure 1. Histograms representative of the occurrence of full bloom in peach per decennial from 2007 to 2012 (A) and in 2013 (B) in the peach collection of the Experimental Station of Urussanga, located in the southern state of Santa Catarina. The graphs are the result of 783 and 266 phenological records, respectively

Tabela 2. Número de horas com temperatura entre 13,0 e $16,0^{\circ} \mathrm{C}$ acumuladas a cada dez dias a partir de 1 ㅇ de janeiro, nos anos de 2007 a 2016, em Urussanga, SC Table 2. Number of hours with temperature between 13.0 and $16.0^{\circ} \mathrm{C}$ accumulated every ten days from January 1, 2007 to 2016, in Urussanga, SC

\begin{tabular}{ccccccccccc}
\hline Ano & $\begin{array}{c}\text { Até } \\
10 / 01\end{array}$ & $\begin{array}{c}\text { Até } \\
20 / 01\end{array}$ & $\begin{array}{c}\text { Até } \\
31 / 01\end{array}$ & $\begin{array}{c}\text { Até } \\
10 / 02\end{array}$ & $\begin{array}{c}\text { Até } \\
20 / 02\end{array}$ & $\begin{array}{c}\text { Até } \\
28 / 02\end{array}$ & $\begin{array}{c}\text { Até } \\
10 / 03\end{array}$ & $\begin{array}{c}\text { Até } \\
20 / 03\end{array}$ & $\begin{array}{c}\text { Até } \\
31 / 03\end{array}$ & $\begin{array}{c}\text { Até } \\
10 / 04\end{array}$ \\
\hline 2007 & 0 & 4 & 5 & 6 & 8 & 8 & 8 & 10 & 10 & 10 \\
2008 & 0 & 0 & 0 & 9 & 9 & 9 & 9 & 11 & 14 & 34 \\
2009 & 14 & 14 & 18 & 18 & 20 & 20 & 20 & 21 & 21 & 29 \\
2010 & 0 & 0 & 0 & 0 & 0 & 5 & 5 & 6 & 6 & 39 \\
2011 & 0 & 0 & 0 & 0 & 0 & 0 & 1 & 11 & 18 & 48 \\
2012 & 1 & 1 & 3 & 3 & 3 & 3 & 3 & 6 & 24 & 39 \\
2013 & 1 & 1 & 6 & 6 & 6 & 6 & 7 & 15 & 24 & 33 \\
2014 & 3 & 3 & 3 & 3 & 3 & 3 & 3 & 3 & 19 & 19 \\
2015 & 0 & 0 & 0 & 0 & 0 & 0 & 0 & 0 & 0 & 15 \\
2016 & 0 & 0 & 0 & 0 & 0 & 0 & 1 & 3 & 5 & 5 \\
\hline
\end{tabular}

Fonte: EPAGRI /CIRAM (2018)

Tabela 3. Número de horas com temperatura entre 16,0 e $19,0^{\circ} \mathrm{C}$ acumuladas a cada dez dias a partir de 10 de janeiro, nos anos de 2007 a 2016, em Urussanga, SC Table 3. Number of hours with temperature between 16.0 and $19.0^{\circ} \mathrm{C}$ accumulated every ten days from January 1st, in the years 2007 to 2016, in Urussanga, SC

\begin{tabular}{ccccccccccc}
\hline \multirow{2}{*}{ Ano } & $\begin{array}{c}\text { Até } \\
10 / 01\end{array}$ & $\begin{array}{c}\text { Até } \\
20 / 01\end{array}$ & $\begin{array}{c}\text { Até } \\
31 / 01\end{array}$ & $\begin{array}{c}\text { Até } \\
10 / 02\end{array}$ & $\begin{array}{c}\text { Até } \\
20 / 02\end{array}$ & $\begin{array}{c}\text { Até } \\
28 / 02\end{array}$ & $\begin{array}{c}\text { Até } \\
10 / 03\end{array}$ & $\begin{array}{c}\text { Até } \\
20 / 03\end{array}$ & $\begin{array}{c}\text { Até } \\
31 / 03\end{array}$ & $\begin{array}{c}\text { Até } \\
10 / 04\end{array}$ \\
\hline 2007 & 5 & 20 & 33 & 45 & 94 & 94 & 94 & 111 & 115 & 143 \\
2008 & 9 & 12 & 51 & 77 & 85 & 86 & 88 & 139 & 156 & 212 \\
2009 & 50 & 70 & 98 & 108 & 124 & 124 & 124 & 152 & 178 & 214 \\
2010 & 0 & 4 & 4 & 4 & 5 & 25 & 38 & 57 & 67 & 143 \\
2011 & 0 & 0 & 0 & 0 & 1 & 2 & 27 & 75 & 96 & 171 \\
2012 & 34 & 36 & 65 & 65 & 77 & 77 & 84 & 114 & 169 & 227 \\
2013 & 24 & 63 & 93 & 119 & 119 & 132 & 163 & 239 & 303 & 327 \\
2014 & 21 & 22 & 24 & 24 & 35 & 41 & 63 & 65 & 96 & 107 \\
2015 & 2 & 2 & 12 & 19 & 21 & 26 & 37 & 52 & 83 & 145 \\
2016 & 0 & 8 & 24 & 24 & 24 & 34 & 61 & 87 & 132 & 132 \\
\hline Fonte: EPAGRI/CIRAM (2018) & & & & & & & &
\end{tabular}


seleção avançada de pessegueiros (seleção 205) que apresentou floração precoce em abril de 2013. Através desta figura, é possível observar que as temperaturas abaixo de $19,0^{\circ} \mathrm{C}$ acumuladas entre 11 de fevereiro e 31 de março estão significativamente correlacionadas com a data de plena floração $(R=-0,85$; $p<0,02)$. No gráfico também pode ser observado que as temperaturas entre 16,0 e $19,0^{\circ} \mathrm{C}$ constituem a principal faixa térmica relacionada com esta antecipação $(R=-0,92 ; p<0,01)$. Outros acessos que registraram floração em abril de 2013 apresentaram resultados semelhantes aos da Figura 2 (dados não apresentados).

Os modelos de unidades de frio tradicionais não consideram as temperaturas entre 16,0 e $19,0^{\circ} \mathrm{C}$ como efetivas para a necessidade de resfriamento das gemas durante a endodormência (HARRINGTON et al., 2010; MELKE, 2015). Enquanto modelos como o de Utah (RICHARDSON et al., 1974) e da Carolina do Norte (SHALTOUT \& UNRATH, 1983) consideram que esta faixa térmica contribui negativamente para o frio acumulado, outros modelos como o de horas de frio ponderadas (EREZ \& LAVEE, 1971; CARDOSO et al., 2015) e o "Low Chilling Model" (GILREATH \& BUCHANAN, 1981) não consideram nenhum efeito. Neste último modelo, as temperaturas entre 17,0 e $19,4^{\circ} \mathrm{C}$ apresentam um valor de unidade de frio igual a zero e, portanto, é considerado que as temperaturas entre 16,0 e $17,0^{\circ} \mathrm{C}$ contribuem positivamente para a acumulação de frio, embora com menor efetividade que as temperaturas inferiores.

Segundo Mahhou e Dennis (1995), sementes e gemas de pessegueiros com baixa exigência em frio podem apresentar uma faixa mais ampla de temperaturas com efeitos vernalizantes. Fuchigami et al. (1982) consideraram, em seu modelo, que temperaturas entre $-3,5$ e $21,0^{\circ} \mathrm{C}$ são efetivas como unidades de frio para a quebra da dormência. Considerando esta faixa, as temperaturas entre 16,0 e $19,0^{\circ} \mathrm{C}$ poderiam ser as principais responsáveis pela antecipação do florescimento observada, em razão de seu efeito vernalizante.

Muitos modelos fenológicos sustentam que as plantas satisfazem sequencialmente as suas necessidades de frio e calor para o florescimento/

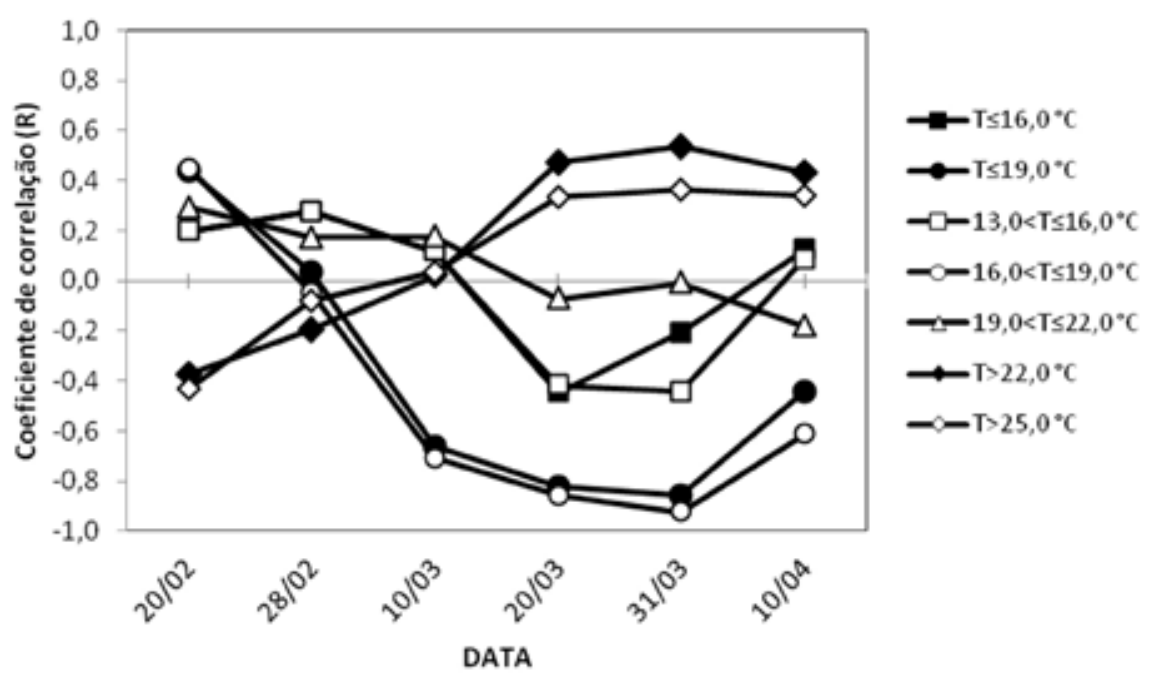

Figura 2. Coeficientes de correlação entre a data de plena floração da 'seleção 205' de pessegueiro e os totais de horas, acumulados de 11/02 até determinadas datas (DATA), em que a temperatura ( $T$ ) permaneceu em determinados níveis térmicos. Urussanga-SC, 2008 a 2014

Figure 2. Correlation coefficients between the full bloom date of peach selection 205 and total accumulated hours of 11/02 until certain dates (DATA), where the temperature (T) remained at certain thermal levels. Urussanga-SC, 2008 to 2014

brotação. Outros trabalhos e estudos, entretanto, consideram que os acúmulos de frio e de calor podem ocorrer de forma paralela ou alternada (FISHMAN et al., 1987; CAMPOY et al., 2011; OKIE \& BLACKBURN, 2011b; HARRINGTON \& GOLD, 2015; POLA et al., 2016). No presente estudo, pode-se apenas hipotetizar sobre como o calor atuou nas plantas em 2013. Sabe-se somente que a interação entre o frio acumulado e o calor disponível foi suficiente para promover a plena floração em meados de abril. Se for considerado que, até o dia 31 de março, as gemas utilizaram as baixas temperaturas disponíveis $(13,0$ a $19,0^{\circ} \mathrm{C}$ ) somente para a liberação da endodormência (Figura 2), teríamos, em um modelo sequencial, a influência do calor ocorrendo após a acumulação do frio, de 10 de abril até a data da plena floração (15 de abril), por exemplo. Entretanto, se for considerado um modelo paralelo de acumulação de unidades de frio e de calor, a acumulação de calor pode ter iniciado antes de 31 de março. Neste caso, não é possível afirmar, no presente estudo, se as temperaturas entre 16,0 e $19,0{ }^{\circ} \mathrm{C}$ tiveram realmente um efeito vernalizante ou se serviram apenas para a acumulação de calor.

É comum serem observadas florações erráticas em alguns genótipos de pessegueiro nos meses de maio e junho na região do presente estudo, mas não no mês de abril e nem com a intensidade registrada em 2013. Os resultados obtidos por Okie e Blackburn (2011a) sugerem que as florações erráticas em pessegueiros estão relacionadas com as diferentes necessidades de frio das gemas dentro de uma mesma planta ou ramo. Assim, uma gema de pessegueiro, após ter acumulado frio acima de um determinado nível crítico, poderá responder ao calor disponível, florescendo precocemente. Outras gemas não florescerão por não terem acumulado uma quantidade mínima de frio necessária para poder responder ao calor ou por não terem o calor necessário (ecodormentes).

Individualmente, as gemas vão entrando na endodormência à medida que as inibições correlativas vão diminuindo (por exemplo, à medida que as folhas vão caindo). Como hipótese, temos que, em 2013, a queda precoce e relativamente rápida das folhas em razão da ferrugem provocou também um início antecipado e conjunto de grande número de gemas no estágio de endodormência. O nível da endodormência permaneceu relativamente uniforme e superficial, em razão da faixa de temperaturas vernalizantes estar relativamente elevada e estreita. A interação entre o frio acumulado e o calor disponível 
foi suficiente para promover a abertura destas gemas, resultando em uma floração intensa naqueles acessos com menor exigência de frio.

\section{Conclusões}

Temperaturas de 13,0 a $19,0^{\circ} \mathrm{C}$ apresentaram uma correlação negativa e estatisticamente significativa com a plena floração. Essa faixa térmica foi a que mais se relacionou com a antecipação anômala da plena floração observada no estudo.

O florescimento intenso observado em abril de 2013 aparentemente foi causado pela queda antecipada e relativamente abrupta das folhas em associação com a disponibilidade de baixas temperatura e de calor para a abertura das gemas.

\section{Referências}

BALANDIER, P.; GENDRAUD, M.; RAGEAU, R.; BONHOMME, M.; RICHARD, J.P.; PARISOT, E. Bud break delay on single node cuttings and bud capacity for nucleotide accumulation as parameters for endo- and paradormancy in peach trees in a tropical climate. Scientia Horticulturae, Amsterdam, v.55, n.3-4, p.249-261, 1993.

BALANDIER, P.; RAGEAU, R.; GENDRAUD, M.; BONHOMME, M.; PARISOT, E. Dormancy in peach tree under the tropical climate of Reunion Island: biological and biochemical approaches. Acta Horticulturae, v.409, p.3946, 1995.

BONHOMME, M.; RAGEAU, R.; GENDRAUD, $M$. Influence of temperature on the dynamics of ATP, ADP and non-adenylic triphosphate nucleotides in vegetative and floral peach buds during dormancy. Tree Physiology, Victoria, v.20, p.615-621, 2000.

CAMPOY, J. A.; RUIZ, D.; COOK, N.; ALLDERMAN, L.; EGEA, J. High temperatures and time to budbreak in low chill apricot 'Palsteyn'. Towards a better understanding of chill and heat requirements fulfilment. Scientia Horticulturae, Amsterdam, v.129, n.4, p.649-655, 2011.

CARDOSO, L.S.; BERGAMASCHI, H.; BOSCO, L.C.; DE PAULA, V.A.; NACHTIGAL, G.R. Unidades de frio para macieiras na região de Vacaria-RS, Brasil. Revista Brasileira de Fruticultura, Jaboticabal, v.37, n.2, p.289-295, 2015.

EPAGRI /CIRAM- Empresa de pesquisa agro- pecuária e extensão rural de Santa Catarina s.a / Centro de informações de recursos ambientais e de hidrometeorologia de Santa Catarina. Dados da estação meteorológica de Urussanga - Florianópolis, 2018.

EREZ, A.; LAVEE, S. The effect of climatic conditions on dormancy development of peach buds. I - Temperature. Proceedings of the American Society for Horticultural science, Alexandria, v.96, n.6, p.711-714, 1971.

FISHMAN, S.; EREZ, A.; COUVILLON, G.A. The temperature dependence of dormancy breaking in plants: Mathematical analysis of a two-step model involving a cooperative transition. Journal of Theoretical Biology, Amsterdam, v.124, p.473-483, 1987.

FUCHIGAMI, L. H.; WEISER, C. J.; KOBAYASHI, K. D.; TIMMIS, R.; GUSTA, L. V. A degree growth stage (OGS) model and cold acclimation in temperate woody plants. In: P. H. Li \& A. Sakai (Eds.). Plant cold hardiness and freezing stress: Mechanisms and crop implications Acad. Press, New York, v.2, 1982. p.93-116.

GILREATH, P.R.; BUCHANAN, D.W. rest prediction model for low chilling 'Sungold" nectarine. Journal of the American Society for Horticultural Science, Alexandria, v.106, n.4, p.426-429, 1981.

HARRINGTON, C.A.; GOULD, P.J.; ST CLAIR, B. BRADLEY, J. Modeling the effects of winter environment on dormancy release of Douglas-fir. Forest Ecology and Management, Netherlands, v.259, p.798-808, 2010.

HARRINGTON, C.A.; GOULD, P.J. Tradeoffs between chilling and forcing in satisfying dormancy requirements for Pacific Northwest tree species. Frontiers in Plant Science, Lausanne, v.6, n.120, 2015.

HRUY, G.; TEGENBOS, J.; PETRE, R.; DECKERS, T.; TEKLEBIRHAN, Y.; BAUER, H.; GEBREHIWOT, K.; RAES, D.; DECKERS, J.; KEULEMANS, J. Studies on mode of expression of apple (Malus x domestica Borkh.) bud dormancy under tropical and temperate climatic conditions. Journal of Agricultural Science and Technology B, Tehan, v.3, p.503-516, 2013.

LANG, G.A.; EARLY, J.D.; MARTIN, G.C.; DARNELL, R.L. Endodormancy, paradormancy, and ecodormancy - Physiological terminology and classification for dormancy research. HortScience, Pleasanton, v.22, p.371-377, 1987.

LI, Y.; FANG, W.; ZHU, G.; CAO, K.; CHEN, C.; WANG, X.; WANG, L. Accumulated chilling hours during endodormancy impact blooming and fruit shape development in peach
(Prunus persica L.). Journal of Integrative Agriculture, Zhengzhou, v.15, n.6, p.12671274, 2016.

LLOYD, J.Y.; FIRTH, D.J. Effect of defoliation time on depth of dormancy and bloom time for low-chill peaches. HortScience, Alexandria, v.25, n.12, p.1575-1578, 1990.

MAHHOU, A.; DENNIS, F.G. Effects of constant and alternating temperatures on breaking dormancy of peach (Prunus persica L.) seeds. Actes Inst. Agron. Vet (Maroc), Rabat, v.15, n.1, p.21-26, 1995.

MELKE, A. The physiology of chilling temperature requirements for dormancy release and bud-break in temperate fruit trees grown at mild winter tropical climate. Journal of Plant Studies, Toronto, v.4, n.2, 2015.

OKIE, W.R.; BLACKBURN, B. Increasing chilling reduces heat requirement for floral budbreak in peach. HortScience, Alexandria, v.46, n.2, p.245-252, 2011 a.

OKIE, W.R.; BLACKBURN, B. Interactive effects of light and chilling on peach flower and leaf budbreak. HortScience, Alexandria, v.46, n.7, p.1056-1062, 2011b.

POLA, A.C.; DELLA BRUNA, E.; BACK, A.J.; MORETO, A.L. Influence of different temperature levels on the date of full bloom of peach varieties in subtropical climate. Revista Brasileira de Fruticultura, Jaboticabal, v.38, n.4, 2016.

RICHARDSON, E.A.; SEELEY, S.D.; WALKER, D.R. A model for estimating the completion of rest for 'Redhaven' and 'Elberta' peach trees. HortScience, Alexandria, v.1, p.331332, 1974.

SCARIOTTO, S. Fenologia e componentes de rendimento de pessegueiro em condições subtropicais. 130f. Dissertação (Mestrado em Agronomia)-Universidade Tecnológica Federal do Paraná, Pato Branco, PR, 2011.

SHALTOUT, A.D.; UNRATH, C.R. Rest completion prediction model for Starkrimson Delicious apples. Journal of the American Society for Horticultural Science, Mount Vernon, v.108, n.6, p.957-961, 1983.

SHERMAN, W.B.; LYRENE, P.M. Biannual peaches in the tropics. Fruit Varieties Journal, University Park, v.38, n.2, p.37-39, 1984.

YAMANE, H. Regulation of Bud Dormancy and Bud Break in Japanese Apricot (Prunus mume Siebold \& Zucc.) and Peach [Prunus persica (L.) Batsch]: A Summary of Recent Studies. Journal of the Japanese Society for Horticultural Science, Tokyo, v.83, n.3, 2014. 